\title{
Cigarette smoking habit does not reduce the benefit from first line trastuzumab-based treatment in advanced breast cancer patients
}

\author{
DANIELE SANTINI ${ }^{1}$, BRUNO VINCENZI ${ }^{1}$, VINCENZO ADAMO ${ }^{2}$, RAFFAELE ADDEO $^{3}$, VITTORIO FUSCO ${ }^{4}$, \\ ANTONIO RUSSO ${ }^{5}$, FILIPPO MONTEMURRO ${ }^{6}$, ILARIA ROATO ${ }^{7}$, STEFANIA REDANA ${ }^{6}$, \\ GAETANO LANZETTA ${ }^{8}$, MARIA ANTONIETTA SATOLLI ${ }^{9}$, ALFREDO BERRUTI ${ }^{10}$, VALENTINA LEONI ${ }^{1}$, \\ SARA GALLUZZO ${ }^{1}$, MAURO ANTIMI $^{11}$, GIUSEPPA FERRARO ${ }^{2}$, MAURA ROSSI $^{4}$, SALVATORE DEL PRETE ${ }^{3}$, \\ MARIA ROSARIA VALERIO ${ }^{5}$, MONICA MARRA ${ }^{12}$, MICHELE CARAGLIA $^{12}$ and GIUSEPPE TONINI ${ }^{1}$ \\ ${ }^{1}$ Medical Oncology, University Campus Bio-Medico, Rome; ${ }^{2}$ Medical Oncology and Integrated Therapies Unit, \\ University Policlinic ‘G. Martino' of Messina, Messina; ${ }^{3}$ Oncology Unit, S. Giovanni di Dio Hospital, Naples; \\ ${ }^{4}$ Oncology Unit, Azienda Ospedaliera di Alessandria, Alessandria; ${ }^{5}$ Section of Medical Oncology, \\ Department of Surgery and Oncology, University of Palermo, Palermo; ${ }^{6}$ Division of Medical Oncology, \\ Istituto per la Ricerca e la Cura del Cancro, Candiolo; ${ }^{7}$ A.O.U. San Giovanni Battista, Torino; ${ }^{8}$ Oncology Unit, \\ INI Grottaferrata, Rome; ${ }^{9}$ Biomedical Sciences and Oncology, University of Torino; ${ }^{10}$ Medical Oncology, \\ Azienda Ospedaliera Universitaria San Luigi di Orbassano, Torino; ${ }^{11}$ Medical Oncology Ospedale S. Eugenio, Rome; \\ ${ }^{12}$ Department of Biofisica and Biochimica 'Francesco Cedrangolo' Second University of Naples, Naples, Italy
}

Received October 15, 2009; Accepted November 27, 2009

DOI: $10.3892 /$ or.2011.1235

\begin{abstract}
Many ErbB2-positive cancers may show intrinsic resistance, and the frequent development of acquired resistance to ErbB-targeted agents represents a substantial clinical problem. The constitutive NF-кB activation in some HER-2/ neu positive breast cancer may represent a potential cause of resistance to trastuzumab therapy. Preclinical data revealed that 4-( $N$-Methyl- $N$-nitrosamino)-1-(3-pyridyl)-1-butanone (NNK), the tobacco-specific nitrosamine is able to enhance NF-кB DNA binding activity and theoretically to increase the resistance to trastuzumab. Two hundred and forty-eight women with pathologically confirmed, uni- or bidimensionally measurable, HER-2-positive metastatic breast cancer (MBC) treated with trastuzumab-based therapy as first line combination for metastatic disease were considered eligible.
\end{abstract}

Correspondence to: Dr Daniele Santini, Department of Medical Oncology, University Campus Bio-Medico, Rome, Italy

E-mail: d.santini@unicampus.it

Dr Michele Caraglia, Department of Biochemistry and Biophysics, Second University of Naples, Via Costantinopoli, I-16 80138 Naples, Italy

E-mail: michele.caraglia@unina2.it

Abbreviations: $\mathrm{MBC}$, metastatic breast cancer; RR, response rate; TTP, time to progression; OS, overall survival; NF- $\mathrm{KB}$, nuclear factor-кB

Key words: trastuzumab, smoking, metastatic breast cancer
For all included patients data on smoking habit were detectable from medical records. We retrospectively analysed the smoking habits of $248 \mathrm{MBC}$ patients and correlated these habits with activity and efficacy of trastuzumab-based therapy. No statistically significant difference in terms of response rate (RR), time to progression (TTP) and overall survival (OS) was identified between smokers (former plus active smokers) and never smokers. Moreover, no statistically significant difference in terms of RR, TTP and OS was identified either comparing active smokers and former smokers. Moreover, we did not observed any significant statistical difference in terms of TTP and OS between smokers $\geq 10$ cigarettes/day and $<10$ cigarettes/day. This study clearly showed lack of any correlation between cigarette smoking habit and both activity and efficacy of trastuzumab-based first line therapy in metastatic HER2/neu positive breast cancer patients.

\section{Introduction}

There are four members of the EGFR family of receptors, each of which is capable of forming heterodimers with other family members. These members are EGFR (erbB1, HER1), HER2 (erbB2/neu), erbB3 (HER3), and erbB4 (HER4).

The discovery that some women with early-stage breast cancer overexpress HER2 has led to the development of the recombinant humanized monoclonal antibody trastuzumab (Herceptin ${ }^{\circledR}$; Genentech, San Francisco, CA). Trastuzumab is actually indicated for patients with tumors that overexpress HER 2 and has an excellent therapeutic index, with consistent evidence that it improves the efficacy of available therapies in all stages of breast cancer, both in adjuvant and in metastatic settings $(1,2)$. 
However, many ErbB2-positive cancers may show intrinsic resistance, and the frequent development of acquired resistance to ErbB-targeted agents represents a substantial clinical problem. There are many potential mechanisms for resistance to this type of therapy, including the formation of alternative ErbB signaling complexes and crosstalk with other pathways (3).

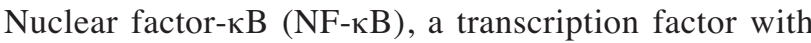
pleotropic effects, is a downstream mediator of growth signaling in erbB2 (HER-2/neu) receptor-positive cancer and activation of NF- $\mathrm{KB}$ in human breast cancer is confined predominately to those that express members of the EGF family of receptors, including the EGF receptor (erbB1) and erbB2 (4). The constitutive NF-kB activation in some HER-2/neu positive breast cancer may represent a potential cause of resistance to trastuzumab therapy (5). Infact, preliminary results also revealed that specific inhibitors of NF- $\mathrm{kB}$ and the EGFR family of cell surface receptors in combinations have demonstrated to block proliferation synergistically at concentrations which are ineffective when used individually (6). Preclinical data revealed that 4-( $N$-Methyl- $N$-nitrosamino)1-(3-pyridyl)-1-butanone (NNK), the tobacco-specific nitrosamine, was able to stimulate the proliferation of SW1116 colon cancer cell line enhancing NF- $\mathrm{KB}$ DNA binding activity (7). Moreover, nicotine, the major alcaloid in tobacco, increases the oxidative stress of colon cancer cells, leading to the activation of NF- $\mathrm{KB}$, a redox-sensitive trascription factor (8) and promotes colon cancer cell proliferation and tumor growth in a dose-dependent manner, increasing EGFR phosphorylation levels (9).

Drawing from the above considerations, our group has evaluated the impact of cigarettes smoking on trastuzumab efficacy in a large cohort of metastatic breast cancer patients treated with trastuzumab-based regimens as first line of therapy.

\section{Materials and methods}

Women with pathologically confirmed, uni- or bidimensionally measurable, HER-2-positive metastatic breast cancer (MBC) not treated with any drug for metastatic disease were considered eligible. Patients were required to be $\geq 18$ years of age, have an Eastern Cooperative Oncology Group performance status of $0-2$. Patients with samples scoring $3+$ by IHC or positive by FISH were eligible, whereas those with a 2+ IHC score also had to have a positive result by FISH. Patients who had received prior chemotherapy for MBC were excluded although prior adjuvant or neoadjuvant chemotherapy was permitted. Concurrent chemotherapy or hormonal therapy was allowed. Patients with brain metastasis not responding to treatment were excluded. At least 4 weeks were required to elapse between last surgery or radiation therapy with full recovery, and indicator lesions had to be outside prior radiation fields.

We considered all consecutive patients for whom data on smoking habit were detectable from medical records between 2004 and 2007 in the following hospitals: Campus BioMedico University of Rome; Azienda Ospedaliera Universitaria San Luigi di Orbassano; S. Giovanni di Dio Hospital of Frattamaggiore; Center for Experimental Research and
Medical Studies San Giovanni Battista, Turin; Section of Medical Oncology, Università di Palermo; Polo Oncologico Alessandria-Asti; Unità Funzionale di Oncologia, INI, Grottaferrata; Medical Oncology, Department of Human Pathology, University Hospital G. Martino, Messina; Divisione di Oncologia Medica, Istituto per la Ricerca e la Cura del Cancro, Candiolo, Torino; and Oncologia Medica, Ospedale S. Eugenio, Rome.

Treatment plan. Trastuzumab was administered according to different schedules (weekly or three-weekly, after induction) and in association with different cytotoxic agents. Tumor response was evaluated every 9 weeks using consistent imaging techniques (CT or MRI). Response assessment was performed by the investigators according to the Response Evaluation Criteria in Solid Tumors (RECIST).

Statistical analysis. We conducted the present cohort study to evaluate whether active or passive cigarettes smoking status affects time to progression (TTP) and overall survival (OS) among MBC patients treated with chemotherapy plus cetuximab. TTP was calculated from the date of starting trastuzumab-based treatment to the first observation of disease progression or to death from any cause, occurring within 60 days after the start of treatment or the most recent tumor assessment. OS time was calculated from the date of starting trastuzumab-based treatment until death from any cause or until the date of the last follow-up, at which point data were censored. The Kaplan-Meier product-limit method was used for analysis of TTP and OS (10).

Moreover, the differences in terms of TTP and OS between different groups were evaluated by the log-rank test (11). The cut-off point for survival data was July 2008; for safety data, it was March 2007. SPSS software (version 13.05, SPSS, Chicago, IL) was used for statistical analysis. A P $<0.05$ was considered to indicate statistical significance.

\section{Results}

We retrospectively analysed the smoking habits of $248 \mathrm{MBC}$ patients (median age 56 years, range $28-86$ years) treated with different anticancer regimens (paclitaxel- and docetaxel-based: 210 patients; vinorelbine-based: 27 patients; anthracyclinebased; 5 patients; carboplatin-based: 6 patients) plus trastuzumab. Table I lists the patient characteristics.

We characterized the following three groups: never smokers, former smokers (patients who had given up smoking at least 6 months before the beginning of trastuzumab-based treatment) and current smokers. Moreover, we further divided the last group in two sub-populations: patients smoking $\leq 10, \geq 10$ cigarettes per day. Data on passive smoking were collected. A passive smoker was defined as a person who usually breaths ambient air containing carcinogenic inhalants from an 'active' cigarette smoker at least for $2 \mathrm{~h}$ every day for not less than 10 years. We did not analyse cigar or pipe smoking.

As reported in Table I our population was composed of 169 never smokers, 33 former smokers and 46 active smokers, (20 $\leq 10$ cigarettes/day, $26>10$ cigarettes/day). We included in this observational trial 59 passive smokers. There were no 
Table I. Patient characteristics.

\begin{tabular}{|c|c|}
\hline Number & 248 \\
\hline \multicolumn{2}{|l|}{ Gender } \\
\hline Male & $3 \quad(1.2 \%)$ \\
\hline Female & $245(98.8 \%)$ \\
\hline \multicolumn{2}{|l|}{ Age (years) } \\
\hline Median & 56 \\
\hline Range & $28-86$ \\
\hline \multicolumn{2}{|l|}{ Histotype } \\
\hline Ductal & $87.1 \%$ \\
\hline Lobular & $10.1 \%$ \\
\hline Other & $2.8 \%$ \\
\hline \multicolumn{2}{|l|}{ Grading } \\
\hline G1 & $8 \%$ \\
\hline G2 & $27 \%$ \\
\hline G3 & $65 \%$ \\
\hline Never smokers & $169(68.1 \%)$ \\
\hline Former smokers & $33(13.3 \%)$ \\
\hline Active smokers & $46(18.6 \%)$ \\
\hline
\end{tabular}

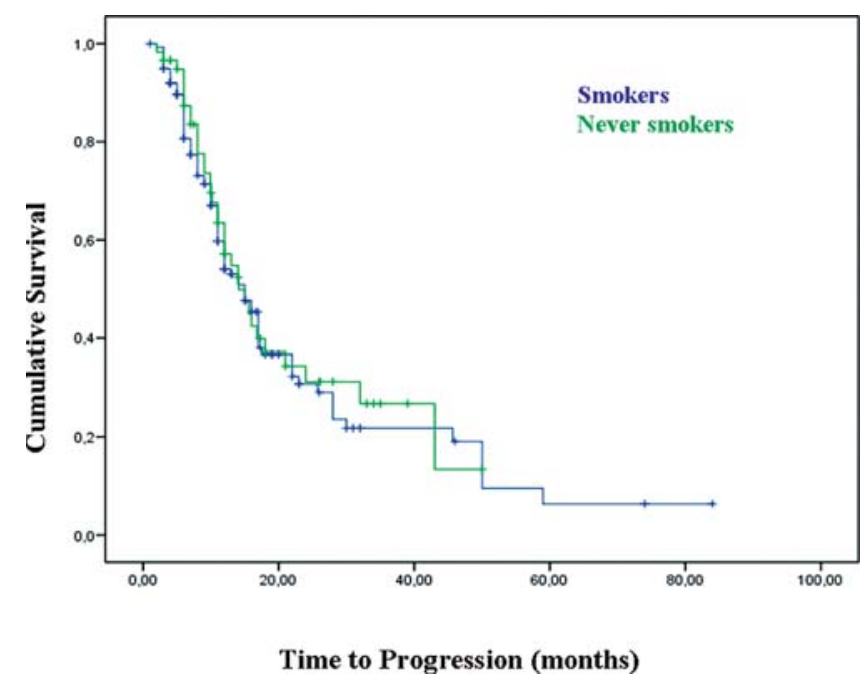

Figure 1. No statistically significant difference in terms of TTP was identified between smokers (former plus active smokers) and never smokers $(\mathrm{P}=0.683)$.

significant differences in terms of age, PS, hormonal receptor status, grading and trastuzumab-associated chemotherapy regimen among the three groups determined by their smoking habit (data not shown).

No statistically significant difference in terms of response rate (RR) and TTP has been identified between smokers (former plus active smokers) and never smokers. The RR in non-smokers was of $50.6 \%$ and in smokers was of $50 \%$ $(\mathrm{P}=0.736)$. Fig. 1 shows the median TTP was 15 months (95\% CI: $12.42-17.58)$ vs. 14.1 (95\% CI: 12.62-17.38) in

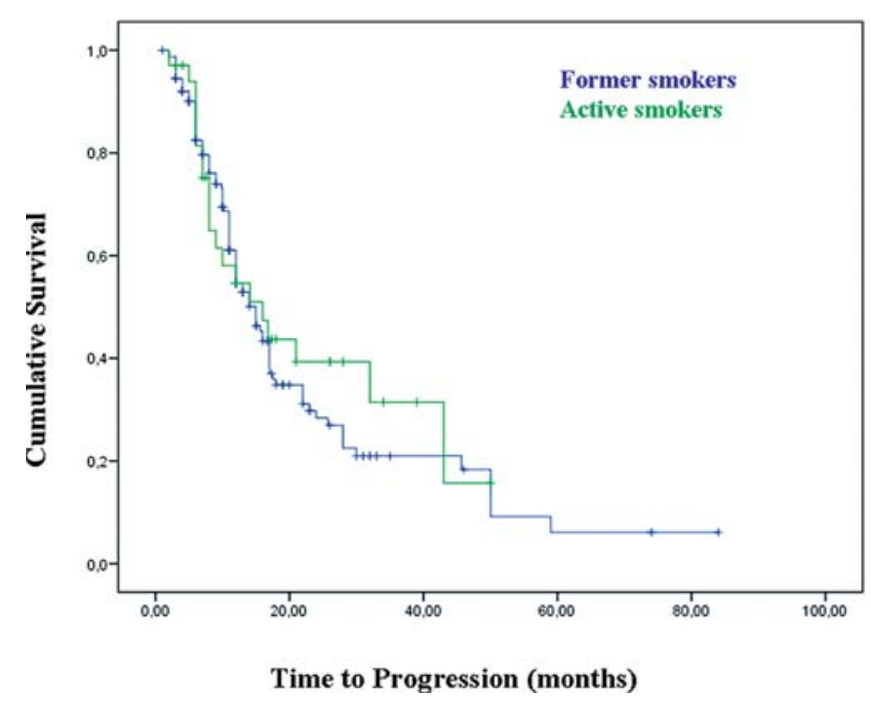

Figure 2. No statistically significant difference in terms of TTP was identified in active or former smokers $(\mathrm{P}=0.581)$.

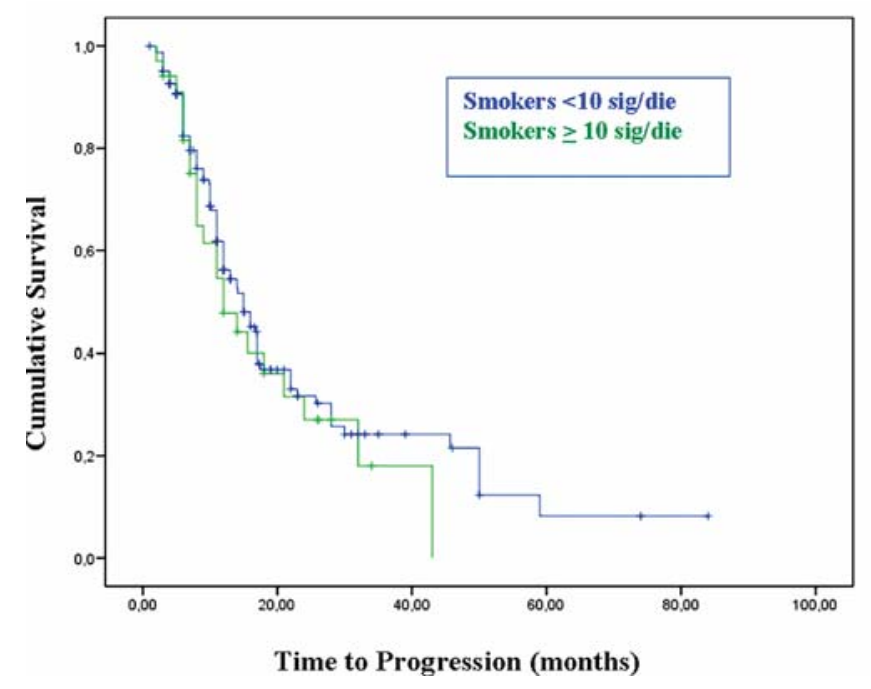

Figure 3. No significant statistical difference was observed in terms of TTP between smokers of $\geq 10$ cigarettes/day or $<10$ cigarettes/day.

non-smokers and smokers, respectively $(\mathrm{P}=0.683)$. Moreover, no statistically significant difference in terms of response rate (RR) and TTP has been identified also comparing active smokers and former smokers. In particular, the RR in former smokers was of 52 versus $42.2 \%$ in active smokers ( $\mathrm{P}=0.462)$. Fig. 2 reports the median TTP was 15 months (95\% CI: 12.54-17.45) vs. 16 (95\% CI: 7.46-24.5) in former smokers and active smokers, respectively $(\mathrm{P}=0.581)$. We did not observe any significant statistical difference in term of TTP between smokers $\geq 10$ cigarettes/day and $<10$ cigarettes/ day, with median values of 12 months (95\% CI: 6.84-17.15) and 15 months (95\% CI: 11.56-18.43), respectively as reported in Fig. 3. In the same subgroups we evaluated the median values of overall survival. Also for overall survival no significant statistical differences have been observed when smokers versus non-smokers $(\mathrm{P}=0.636)$, active smokers and former smokers $(\mathrm{P}=0.431)$ and smokers of $\geq 10$ cigarettes/day 
and $<10$ cigarettes/day $(\mathrm{P}=0.998)$ were compared (survival curves not reported).

\section{Discussion}

This study clearly showed lack of any correlation between cigarette smoking habit and both activity and efficacy of trastuzumab-based first line therapy in metastatic HER2/ neu-positive breast cancer patients. At the same time these results deserve to be confirmed prospectively in other adequately designed clinical trials, given the potentially relevant implications in the clinical practice. Our results could be undermined by the use of retrospectively collected smoking data, rather than data collected at the beginning of trastuzumab therapy.

It must be considered that smoking habits may change following a cancer diagnosis as well as during cancer treatment. Moreover, we did not observe any correlation between cigarette smoking habit and trastuzumab activity and efficacy considering the entire population. The number of hormonal receptor positive patients in our cohort was too low in order to investigate any significant correlation with trastuzumab activity in this subpopulation. Some additional biases should be taken in consideration and limit the reproducibility of the results presented in the present report. Data on smoking habit in our patients population were, at least partially, obtained retrospectively, performing a direct question to patients during a medical visit of by a phone call. This methodology could led to a selection bias excluding deceased patients possibly with a worst prognosis. This point could represent the reason of the longer TTP and OS observed in our analysis when compared with that observed with the same cancer population in published clinical trials (9). On the contrary the observed response rate in the population of smokers vs. nonsmokers is reasonably reliable.

However, despite the constitutive NF- $\mathrm{BB}$ activation in some HER-2/neu positive breast cancer is a frequent genetic condition (4-7) and it has been demonstrated that nicotine has a proliferative role on cancer cells through the PI3K/AKT pathway activation and the PTEN pathway inhibition $(8,9)$, we may suppose that the potential role of nicotine in inducing resistance to trastuzumab therapy in $\mathrm{MBC}$ is overcome by other predominant molecular and/or genetic mechanisms conditioning the proliferation and invasive processes of cancer in the metastatic phase. With the same rational, the adjuvant setting of breast cancer appears to represent the best setting to analyse the potential detrimental effects of nicotine in trastuzumab based adjuvant therapy.
In conclusion, despite the potential methodological limits of the present retrospective study, the large number of included patients and the very accurate modality of cigarette habit data collection, allow us to strongly suggest the lack of any activity and efficacy interaction between smoke and trastuzumab based therapy in MBC.

\section{References}

1. Piccart-Gebhart MJ, Procter M, Leyland-Jones B, Goldhirsch A, Untch M, Smith I, Gianni L, et al: Trastuzumab after adjuvant chemotherapy in HER2-positive breast cancer. N Engl J Med 353: 1659-1672, 2005.

2. Slamon DJ, Leyland-Jones B, Shak S, Fuchs H, Paton V, Bajamonde A and Biswas DK: Use of chemotherapy plus a monoclonal antibody against HER2 for metastatic breast cancer that overexpresses HER2. N Engl J Med 344: 783-792, 2001.

3. Piccart M: Circumventing de novo and acquired resistance to trastuzumab: new hope for the care of ErbB2-positive breast cancer. Clin Breast Cancer 3: S100-S113, 2008.

4. Biswas DK, Shi Q, Baily S, Strickland I, Ghosh S, Pardee AB and Iglehart JD: NF-kappa B activation in human breast cancer specimens and its role in cell proliferation and apoptosis. Proc Natl Acad Sci USA 101: 10137-10142, 2004.

5. Singh S, Shi Q, Bailey ST, Palczewski MJ, Pardee AB, Iglehart JD and Biswas DK: Nuclear factor-kappaB activation: a molecular therapeutic target for estrogen receptor-negative and epidermal growth factor receptor family receptor-positive human breast cancer. Mol Cancer Ther 6: 1973-1982, 2007.

6. Biswas DK and Iglehart JD: Linkage between EGFR family receptors and nuclear factor kappaB (NF-kappaB) signaling in breast cancer. J Cell Physiol 209: 645-652, 2006.

7. Ye YN, Liu ES, Shin VY, Wu WK and Cho CH: The modulating role of nuclear factor-kappaB in the action of alpha7-nicotinic acetylcholine receptor and cross-talk between 5-lipoxygenase and cyclooxygenase- 2 in colon cancer growth induced by $4-(\mathrm{N}$ methyl-N-nitrosamino)-1-(3-pyridyl)-1-butanone. J Pharm Exp Ther 311: 123-130, 2004.

8. Crowley-Weber CL, Dvorakova K, Crowley C, Bernstein H, Bernstein C, Garewal $\mathrm{H}$ and Payne $\mathrm{CM}$ : Nicotine increases oxidative stress, activates NF-kappaB and GRP78, induces apoptosis and sensitizes cells to genotoxic/xenobiotic stresses by a multiple stress inducer, deoxycholate: relevance to colon carcinogenesis. Chem Biol Interact 145: 53-66, 2003.

9. Ye YN, Liu ES, Shin VY, Wu WK, Luo JC and Cho CH: Nicotine promoted colon cancer growth via epidermal growth factor receptor, c-Src, and 5-lipoxygenase-mediated signal pathway. J Pharm Exp Ther 308: 66-72, 2004.

10. Kaplan E and Meier P: Non parametric estimation from incomplete observations. J Am Statist Assoc 53: 457-481, 1958.

11. Peto R, Pike MC, Armitage P, Breslow NE, Cox DR, Howard SV, Mantel N, McPherson K, Peto J and Smith PG: Design and analysis of randomized clinical trials requiring prolonged observation of each patient. II. analysis and examples. Br J Cancer 35: 1-39, 1977. 